\section{Results from a Bench Marking Survey on Cassette Dosing Practices in the Pharmaceutical Industry}

Bradley L. Ackermann

Lilly Research Laboratories

Eli Lilly and Company

Indianapolis, Indiana

This communication provides a report of the Pharmaceutical Interest Group Workshop held on Tuesday, June 10th, 2003, during the 51st ASMS Conference on Mass Spectrometry and Allied Topics in Montreal, Canada. The subject of the workshop, cassette dosing (CD), was selected to address two objectives. The first objective was to demonstrate the capacity the ASMS Pharmaceutical Interest Group to serve as a conduit for benchmark analysis on a topic of pharmaceutical interest. A secondary motive was to use the workshop to provide a forum for active discussion and debate. CD is a topic that met both objectives since it is a controversial subject for which no clear consensus exists within the pharmaceutical industry regarding it use.

As anticipated, the topic of CD sparked lively discussion from the nearly 150 society members in attendance. To facilitate bench marking, an informal survey on $\mathrm{CD}$ practices was conducted prior to the meeting. The results from this survey, which were presented at the workshop, are the subject of the present communication.

The beginning of the workshop was devoted to general business including the dissemination of information about the ASMS Pharmaceutical Interest Group. As this information will not be reviewed here, inquiries should be directed to the current chair, Brad Ackermann (ackermann_bradley_1@lilly.com).

\section{Background on Cassette Dosing}

Cassette dosing (a.k.a. N-in-one) refers to the practice of simultaneously dosing laboratory animals with multiple compounds, typically to accelerate the pace of exposure screening. This practice, made possible by the extraordinary specificity of tandem MS detection, is seen as a way to maximize the utilization of expensive LC/MS/MS instrumentation and to minimize the number of animals used. Since the initial reporting of $\mathrm{N}$-in-one dosing by Berman and co-workers in 1997 [1], several reports have appeared in the literature [2-5]. Interested readers are also referred to a seminal article by White and Manitpisitkul on the pharmacokinetic implications of CD [6].

Published online August 6, 2004

Address reprint requests to Dr. B. L. Ackermann, Lilly Research Laboratories, Eli Lilly and Company, Lilly Corporate Center, Indianapolis, IN 46285 , USA. E-mail: ackermann_bradley_1@lilly.com
Two speakers were invited to give short presentations at the workshop on their experience with CD. Cornelius Hop (Pfizer, Groton, CT) shared information related to the consistency of a reference compound dosed in both I.V. and P.O. routes over the course of 45 experiments. Although the results were fairly consistent, differences as high as 5-fold were observed for several PK parameters.

Jing-Tao Wu (Millennium Pharmaceuticals, Cambridge, MA) provided an overview of the use of CD in his laboratory, which included a comparison of single compound and cassette administration $(\mathrm{N}=5)$ for 28 compounds. The half-life values (T 1/2) obtained by the two methods were compared to see how often results produced by CD were outside of the variation explained by single compound administration. Outliers were identified as values $\pm 4 \sigma$ from the mean value observed from single compound administration. The outcome of this experiment was that $75 \%$ of the compounds studied exhibited no change, $21.4 \%$ were considered false positives, and $3.6 \%$ were classified as false negatives. Because false positives are not removed from future screening, the risk of cassette dosing $(\mathrm{N}=5)$ was taken as $3.6 \%$. This risk was deemed acceptable by the author since a 2.5-fold increase in the rate of exposure screening was achieved using the practices described.

\section{Questionnaire on Cassette Dosing Practices}

The remainder of the workshop was devoted to the questionnaire, which consisted of 13 multiple-choice questions. The primary group targeted was bioanalytical experts having either direct experience with CD or an informed opinion on the subject. Several questionnaires were sent both within and outside the United States yielding a total of 37 responses. Of these, "Large Pharma", defined as companies with more than 15,000 employees, accounted for 70 percent of the total responses representing 12 major pharmaceutical companies. The percentage of responses from "Small Pharma/ Biotechs" was 16 percent. Contract research organizations (CROs) accounted for 11 percent of the total responses. When the respondents were asked about their work function, 60 percent indicated they support drug discovery ADME compared with 33 percent who support either preclinical (GLP) or clinical bioanalysis.

The results of the questionnaire were presented in sequential order allowing time for group discussion after each question. The questionnaire is reproduced below. The responses to each question are listed in parentheses. 
QUESTION 1: Describe the use of cassette dosing at your facility
(a) Never $(25 \%)$
(b) Seldom-according to project or upon request $(51 \%)$
(c) Approximately equal to single compound administration based on the number of doses $(16 \%)$
(d) Default method used for exposure screening $(8 \%)$

QUESTION 2: What is the average number of compounds per cassette?
(a) $\mathrm{N}=3$ to $4(52 \%)$
(b) $\mathrm{N}=5$ to $7(41 \%)$
(c) $\mathrm{N}=8$ to $10(7 \%)$
(d) $\mathrm{N}>10(0 \%)$

QUESTION 3: Which species have you employed for cassette dosing?
(a) Mouse (17\%)
(b) Rat $(59 \%)$
(c) $\operatorname{Dog}(17 \%)$
(d) Monkey $(7 \%)$
(e) Other $(0 \%)$

QUESTION 4: How often are cassettes administered by a route other than P.O.?
(a) $<10 \%(27 \%)$
(b) 10 to $49 \%(23 \%)$
(c) 50 to $74 \%(12 \%)$
(d) $>75 \%(38 \%)$

QUESTION 5: How much do formulation issues influence cassette dosing?
(a) Very little $(51 \%)$
(b) Adds noticeable overhead to setup time $(39 \%)$
(c) P.O. dosing used primarily to minimize formulation issues $(10 \%)$
(d) Cassette dosing not performed in part due to formulation issues $(0 \%)$

QUESTION 6: What best describes the reference compound co-administered with each cassette for a given SAR?
(a) Current lead (subject to change as SAR progresses) $(15 \%)$

(b) A well-characterized compound within the SAR (not subject to change) (39\%)

(c) A well-characterized compound not belonging to the SAR (12\%)

(d) A reference compound is not typically dosed with cassettes $(27 \%)$
QUESTION 7: What MS controls are used with cassette dosing? [Results are normalized to response (a)]
(a) Compounds of the same MW are not co-administered $(100 \%)$
(b) Compounds related by likely me- tabolite shifts are not co-adminis- tered $(64 \%)$
(c) MS/MS cross-talk examined for co-administered compounds $(77 \%)$
(d) An internal standard is used for all analysis $(82 \%)$

QUESTION 8: Cassette dosing extends analysis time (i.e., method development, sample preparation, sample analysis, and data processing) by what factor?
(a) No difference relative to single compound administration (19\%)
(b) 50 percent more time needed for cassette dosing $(54 \%)$
(c) 100 percent more time needed for cassette dosing $(19 \%)$
(d) $>100$ percent more time needed for cassette dosing $(8 \%)$

QUESTION 9: Is extra analytical effort applied when cassette dosing is used?
(a) LC conditions selected to maxi- mize throughput (run time not extended) (32\%)
(b) Fast LC conditions used, but cross-talk avoided (run time not extended) (32\%)
(c) Run time extended, although LC separation not necessary unless cross-talk observed (25\%)
(d) LC resolution ensured for all co- administered compounds (11\%)

QUESTION 10: What is your primary motivation for cassette dosing?

[Numbers in parentheses indicate percentage listed as top response]
(a) Maximize MS utilization and ana- lytical $(2 \%)$
(b) Limit number of animals and an- imal handling $(29 \%)$
(c) Maximize throughput and/or ca- pacity for exposure screening $(69 \%)$
(d) Reduce inter-animal variation $(0 \%)$

QUESTION 11: What is the greatest risk or detraction to cassette dosing?

[Numbers in parentheses indicate percentage listed as top response] 
(a) Likelihood of false positive results-reduced clearance due to drug-drug interactions (67\%)

(b) Chance of false negative results $(18 \%)$

(c) Hard to correlate results with in vitro parameters acquired for individual components (11\%)

(d) Exposure throughput not ratelimiting (expense not justified) $(14 \%)$

QUESTION 12: Has in vitro ADME screening decreased your demand for cassette dosing?

(a) Dramatic decline $(>75 \%)(4 \%)$

(b) Significant decline (26 to $75 \%$ ) $(16 \%)$

(c) Noticeable decrease (10 to $25 \%$ ) $(28 \%)$

(d) No change $(<10 \%)(52 \%)$

QUESTION 13: How would you rate your overall confidence with cassette dosing?

(a) Cassette dosing gives acceptable pharmacokinetic information and should be the default method for exposure screening (9\%)

(b) With proper controls cassette dosing can be to rank order compounds $(64 \%)$

(c) Cassette dosing is justified only when exposure information is rate-limiting to a project (15\%)

(d) Advantages do not out-weigh risks - cassette dosing should not be performed $(12 \%)$

\section{Discussion}

The responses to the survey captured a number of trends, which are discussed below. Although the responses received reflect a broad coverage of the pharmaceutical industry, no claims can be made regarding the statistical validity of the results reported.

The response to Question 1 revealed that most of the people surveyed do not routinely perform CD. In fact, only $8 \%$ of the respondents use CD as their default for exposure screening. It was further revealed from Question 2 that those laboratories which engage in cassette dosing employ relatively small cassettes. This trend, which obviously reflects a desire to limit drug-drug interactions, is consistent with recommendations made by White and Manitpisitkul [6]. It is also consistent with the responses received to Question 11 on the risks associated with CD.

As predicted, rat was the most widely employed species for CD. Interestingly, almost a quarter of the respondents indicated large animal experience. This finding is perhaps attributed to the need to conserve large animal resources.

Questions 4 and 5 dealt with alternate routes of administration and formulation issues, respectively. Given the importance of CD for exposure screening, it was interesting to find that roughly half of the time a route other than P.O. is used for CD. Given this result, it was somewhat surprising to learn from Question 5 that relatively few people acknowledged issues with formulation, even though CD using I.V. dosing would appear to be a relatively common practice. During the discussion at the workshop it was revealed that some practitioners filter incompletely dissolved dose solutions and perform an analysis to determine the actual I.V. dose delivered. It was not clear how widespread this practice is.

The survey confirmed that the practice of including a reference compound in all cassettes is a routine practice. Nearly half of the respondents use a well-characterized compound from the SAR that is not subject to change as the SAR progresses. It is also noted that $27 \%$ of the respondents do not use a reference compound in the cassette.

Questions 7 through 9 dealt with analytical issues. According to Question 8, just over half of the respondents acknowledged that CD extends analysis time by $50 \%$. This response was qualitatively consistent with the results for Questions 7 and 9, which indicate that rigorous controls over selectivity including the chromatographic resolution of co-administered compounds are often not imposed; otherwise the net increase would have likely been greater.

Question 10 leaves little doubt about the primary motivation for $C D$, which is to accelerate the rate of exposure screening. The second most cited objective is to limit resources needed for animals and animal handling. When asked about the greatest risk of CD, two-thirds of the respondents indicated the risk associated with drug-drug interactions. Interestingly, the potential for false negative results was a secondary concern $(18 \%)$.

Finally, Question 13 revealed that a majority of the respondents believe that $\mathrm{CD}$ can be used to rank order compounds provided proper controls are used. An interesting finding was that $9 \%$ of the respondents use $\mathrm{CD}$ as their default method for exposure screening, while an approximately equal number $(12 \%)$ state that CD should not be applied in any circumstance. These results speak to the polarization that continues to exist in the pharmaceutical industry with regard to the use of CD.

\section{Summary}

Results from a survey on CD, conducted on behalf of the ASMS Pharmaceutical Interest Group, are presented which provide an assessment of current practices used in the pharmaceutical industry. The purpose of the present report is to disseminate this information, in accordance with a key objective of the Pharmaceutical 
Interest Group to act as a conduit for benchmark analysis on topics of pharmaceutical interest. Based the level of interaction achieve at the 2003 workshop, it was decided that a similar format would be used in 2004. The topic selected for benchmark analysis is the support of chemical synthesis by MS. In closing, gratitude is expressed towards all who participated in the 2003 workshop.

\section{Acknowledgments}

The author thanks Cornelis Hop and Jing-Tao $\mathrm{Wu}$ for their presentations at the workshop and Judith Sjoberg (ASMS) for arrangements related to the workshop.

\section{References}

1. Berman, J.; Halm, K.; Adkison, K.; Shaffer, J. Simultaneous Pharmacokinetic Screening of a Mixture of Compounds in the Dog Using API LC/MS/MS Analysis for Increased Throughput. J. Med. Chem. 1997, 40, 827-829.
2. McLoughlin, D. A.; Olah, T.V.; Gilbert, J. D. A Direct Technique for the Simultaneous Determination of 10 Drug Candidates in Plasma by Liquid Chromatography-Atmospheric Pressure Chemical Ionization Mass Spectrometry Interfaced to a Prospekt Solid-Phase Extraction System. J. Pharm. Biomed. Anal. 1997, 15, 1893-1901.

3. Beaudry, F.; LeBlanc, J. Y. C.; Coutu, M.; Brown, N. In Vivo Pharmcokinetic Screening in Cassette Dosing Experiments; the Use of On-Line Prospekt Liquid Chromatography/Atmospheric Pressure Chemical Ionization Tandem Mass Spectrometry Technology in Drug Discovery. Rapid Commun. Mass Spectrom. 1998, 12, 1216-1222.

4. Wu, J. T.; Zeng, H.; Qian, M.; Brogdon, B. L.; Unger, S. E. Direct Plasma Sample Injection in Multiple-Component LC-MS-MS Assays for High-Throughput Pharmacokinetic Screening. Anal. Chem. 2000, 72, 61-67.

5. Zhang, M. Y.; Kerns, E.; McConnell, O.; Sonnenberg-Reines, J.; Zaleska, M. M.; Jacobsen, S. J.; Butera, J.; Kreft, A. Brain and Plasma Exposure Profiling in Early Drug Discovery Using Cassette Administration and Fast Liquid Chromatography-Tandem Mass Spectrometry. J. Pharm. Biomed. Anal. 2004, 4, 359-368.

6. White, R. E.; Manitpisitkul, P. Pharmacokinetic Theory of Cassette Dosing in Drug Discovery Screening. Drug Metab. Disp. 2001, 29, 957-966. 